\title{
Haar type and Carleson Constants
}

\author{
Stefan Geiss $\quad$ Paul F.X. Müller *
}

October 30, 2018

\begin{abstract}
For a collection $\mathcal{E}$ of dyadic intervals, a Banach space $X$, and $p \in$ $(1,2]$ we assume the upper $\ell^{p}$ estimates

$$
\left\|\sum_{I \in \mathcal{E}} x_{I} h_{I} /|I|^{1 / p}\right\|_{L_{X}^{p}}^{p} \leq c^{p} \sum_{I \in \mathcal{E}}\left\|x_{I}\right\|_{X}^{p},
$$

where $x_{I} \in X$ and $h_{I}$ denotes the $L^{\infty}$ normalized Haar function supported on $I$. We determine the minimal requirement on the size of $\mathcal{E}$ so that these estimates imply that $X$ is of Haar type $p$. The characterization is given in terms of the Carleson constant of $\mathcal{E}$.
\end{abstract}

2000 Mathematics Subject Classification: 46B07, 46B20

\section{Introduction}

Let $X$ be a Banach space. We fix a non-empty collection of dyadic intervals $\mathcal{E}$ and assume the upper $\ell^{p}$ estimates

$$
\left\|\sum_{I \in \mathcal{E}} x_{I} \frac{h_{I}}{|I|^{1 / p}}\right\|_{L_{X}^{p}} \leq c\left(\sum_{I \in \mathcal{E}}\left\|x_{I}\right\|_{X}^{p}\right)^{\frac{1}{p}}
$$

for finitely supported $\left(x_{I}\right)_{I \in \mathcal{E}} \subset X$ and some $p \in(1,2]$, where $h_{I}$ is the $L^{\infty}$ normalized Haar function supported on $I$. The consequences for $X$, one may

${ }^{*}$ Research of both authors supported in part by FWF Pr. Nr. P150907-N01. 
draw from (1), depend on the size and structure of the collection $\mathcal{E}$. For instance, if $\mathcal{E}$ is a collection of pairwise disjoint dyadic intervals, then any Banach space satisfies (11), hence it does not impose any restriction on $X$. If, on the other hand, we choose $\mathcal{E}$ to be the collection of all dyadic intervals, then the upper $\ell^{p}$ estimates (11) simply state that $X$ is of Haar type $p$; due to important work of G. Pisier [6] the latter condition is equivalent to certain renorming properties of the Banach space $X$.

In this paper we ask how massive a collection $\mathcal{E}$ has to be so that (1) implies that $X$ is of Haar type $p$. We give the answer to this question in terms of the Carleson constant defined by

$$
\llbracket \mathcal{E} \rrbracket:=\sup _{I \in \mathcal{E}} \frac{1}{|I|} \sum_{J \in \mathcal{E}, J \subseteq I}|J| .
$$

The proof is based on the following well-known dichotomy: either $\mathcal{E}$ can be decomposed into finitely many collections consisting of "almost disjoint" dyadic intervals or $\mathcal{E}$ contains large and densely packed blocks of dyadic intervals, with arbitrary high degree of condensation.

Initially we encountered the problem treated here in connection with our efforts to obtain a vector valued version of E. M. Semenov's characterization of bounded operators rearranging the Haar system. See [7] and [3].

\section{Preliminaries}

In the following we equip the unit interval $[0,1)$ with the Lebesgue measure denoted by $|\cdot|$. Let $\mathcal{D}$ denote the collection of dyadic intervals in $[0,1)$, i.e. $I \in \mathcal{D}$ provided that there exist $m \geq 0$ and $1 \leq k \leq 2^{m}$ such that

$$
I=\left[(k-1) / 2^{m}, k / 2^{m}\right)
$$

and let

$$
\mathcal{D}_{n}:=\left\{I \in \mathcal{D}:|I| \geq 2^{-n}\right\} \quad \text { where } \quad n \geq 0 .
$$

The $L^{\infty}$ normalized Haar function supported on $I \in \mathcal{D}$ is denoted by $h_{I}$, i.e. $h_{I}=-1$ in the right half of $I$ and $h_{I}=1$ on the left half of $I$. By $L_{X}^{p}$, $p \in[1, \infty)$, we denote the space of Radon random variables $f:[0,1) \rightarrow X$ such that

$$
\|f\|_{L_{X}^{p}}=\left(\int_{0}^{1}\|f(t)\|_{X}^{p} d t\right)^{1 / p}<\infty .
$$


Haar type. Given $p \in(1,2]$, a Banach space $X$ is of Haar type $p$ provided that there exists a constant $c>0$ such that

$$
\left\|\sum_{I \in \mathcal{D}} x_{I} \frac{h_{I}}{|I|^{1 / p}}\right\|_{L_{X}^{p}} \leq c\left(\sum_{I \in \mathcal{D}}\left\|x_{I}\right\|_{X}^{p}\right)^{\frac{1}{p}}
$$

for all finitely supported families $\left(x_{I}\right)_{I \in \mathcal{D}} \subset X$. We let $H T_{p}(X)$ be the infimum of all possible $c>0$ as above. The central result concerning Haar type is due to G. Pisier [6] and asserts that Haar type $p$ is equivalent to the fact that $X$ can be equivalently renormed such that the new norm has a modulus of smoothness of power type $p$. For additional information see [1, 5] and the references therein.

Carleson Constants. Let $\mathcal{E} \subseteq \mathcal{D}$ be a non-empty collection of dyadic intervals. The Carleson constant of $\mathcal{E}$ is given by equation (2). Next we define consecutive generations of $\mathcal{E}$ and, using $\llbracket \mathcal{E} \rrbracket$, describe a dichotomy for $\mathcal{E}$ known as the almost disjointification and condensation properties.

We define $G_{0}(\mathcal{E})$ to be the maximal dyadic intervals of $\mathcal{E}$ where maximal refers to inclusion. Suppose, we have already defined $G_{0}(\mathcal{E}), \ldots, G_{p}(\mathcal{E})$, then we form

$$
G_{p+1}(\mathcal{E}):=G_{0}\left(\mathcal{E} \backslash\left(G_{0}(\mathcal{E}) \cup \cdots \cup G_{p}(\mathcal{E})\right)\right) .
$$

Given $I \in \mathcal{D}$, let $I \cap \mathcal{E}=\{J \in \mathcal{E}, J \subseteq I\}$ and put

$$
G_{k}(I, \mathcal{E}):=G_{k}(I \cap \mathcal{E}) \quad \text { for } \quad k \geq 1 .
$$

Assume that $\llbracket \mathcal{E} \rrbracket<\infty$ and that $M$ is the largest integer smaller than $4 \llbracket \mathcal{E} \rrbracket+1$. Then

$$
\mathcal{E}_{i}:=\bigcup_{k=0}^{\infty} G_{M k+i}(\mathcal{E}), \quad 0 \leq i \leq M-1,
$$

consists of almost disjoint dyadic intervals, in the sense that for $I \in \mathcal{E}_{i}$,

$$
\sum_{J \in G_{1}\left(I, \mathcal{E}_{i}\right)}|J| \leq \frac{|I|}{2} \text { and } \sum_{K \in I \cap \mathcal{E}_{i}}|K| \leq 2|I| .
$$

Conversely, if $\llbracket \mathcal{E} \rrbracket=\infty$, then for all $n \geq 1$ and $\varepsilon \in(0,1)$ there exists a $K_{0} \in \mathcal{E}$ that is densely packed in the sense that

$$
\sum_{J \in G_{n}\left(K_{0}, \mathcal{E}\right)}|J| \geq(1-\varepsilon)\left|K_{0}\right| .
$$


Based on this we show in Lemma 3.4 that for any $n$ the span $\left(h_{I}\right)_{I \in \mathcal{E}}$ contains a system of functions, with the same joint distribution as the first $2^{n}$ elements of the Haar basis.

The proof of this basic dichotomy and some of its applications can be found in [4, Chapter 3].

\section{Haar Type and Carleson Constants}

The main results of this note are Theorems 3.1 and 3.2. Combined they give an answer to the question as to which families of dyadic intervals $\mathcal{E}$ will detect whether a Banach space $X$ has Haar type $p$. The answer is a dichotomy: either $\llbracket \mathcal{E} \rrbracket<\infty$, then $\mathcal{E}$ does not detect any Haar type of any Banach space, or $\llbracket \mathcal{E} \rrbracket=\infty$, then $\mathcal{E}$ determines the Haar type $p$ constant exactly, for any Banach space $X$ and each $1<p \leq 2$.

Theorem 3.1. Let $p \in(1,2]$ and $\mathcal{E} \subseteq \mathcal{D}$ be a non-empty collection of dyadic intervals. Then the following statements are equivalent:

(1) A Banach space $X$ is Haar type $p$ if there exists $c>0$ such that for all finitely supported families $\left(x_{I}\right)_{I \in \mathcal{D}} \subset X$ one has

$$
\left\|\sum_{I \in \mathcal{E}} x_{I} \frac{h_{I}}{|I|^{1 / p}}\right\|_{L_{X}^{p}} \leq c\left(\sum_{I \in \mathcal{E}}\left\|x_{I}\right\|_{X}^{p}\right)^{\frac{1}{p}} ;
$$

the infimum over all such $c>0$ coincides with $H_{p}(X)$.

(2) $\llbracket \mathcal{E} \rrbracket=\infty$.

Theorem 3.2. Let $p \in(1,2], \mathcal{E} \subseteq \mathcal{D}$ be a non-empty collection of dyadic intervals, and $X$ be a Banach space which is not of Haar type $p$. Then the following statements are equivalent:

(1) There exists a constant $c>0$ such that for all finitely supported families $\left(x_{I}\right)_{I \in \mathcal{D}} \subset X$ one has

$$
\left\|\sum_{I \in \mathcal{E}} x_{I} \frac{h_{I}}{|I|^{1 / p}}\right\|_{L_{X}^{p}} \leq c\left(\sum_{I \in \mathcal{E}}\left\|x_{I}\right\|_{X}^{p}\right)^{\frac{1}{p}} .
$$


(2) $\llbracket \mathcal{E} \rrbracket<\infty$.

Theorem 3.1 and Theorem 3.2 follow immediately from the following two lemmas (and the obvious fact that there are Banach spaces without Haar type $p$ if $p \in(1,2])$.

Lemma 3.3. Let $p \in(1, \infty), \llbracket \mathcal{E} \rrbracket<\infty$, and $X$ be a Banach space. Then there is a constant $c_{p}>0$, depending at most on $p$, such that one has

$$
\left\|\sum_{I \in \mathcal{E}} x_{I} \frac{h_{I}}{|I|^{1 / p}}\right\|_{L_{X}^{p}} \leq c_{p} \llbracket \mathcal{E} \rrbracket^{1-\frac{1}{p}}\left(\sum_{I \in \mathcal{E}}\left\|x_{I}\right\|_{X}^{p}\right)^{\frac{1}{p}}
$$

for all finitely supported $\left(x_{I}\right)_{I \in \mathcal{E}} \subset X$.

Proof. Using (3), we write $\mathcal{E}=\mathcal{E}_{0} \cup \cdots \cup \mathcal{E}_{M-1}$ with $M<4 \llbracket \mathcal{E} \rrbracket+1$ such that the collections $\left\{A_{I}: I \in \mathcal{E}_{i}\right\}$ of pairwise disjoint and measurable sets defined by

$$
A_{I}:=I \backslash \bigcup_{J \in G_{1}\left(I, \mathcal{E}_{i}\right)} J, \quad I \in \mathcal{E}_{i}
$$

satisfy

$$
\frac{1}{2}|I| \leq\left|A_{I}\right| \leq|I|
$$

Since

$$
\begin{aligned}
\left\|\sum_{I \in \mathcal{E}} x_{I} \frac{h_{I}}{|I|^{1 / p}}\right\|_{L_{X}^{p}} & \leq \sum_{i=0}^{M-1}\left\|\sum_{I \in \mathcal{E}_{i}} x_{I} \frac{h_{I}}{|I|^{1 / p}}\right\|_{L_{X}^{p}} \\
& \leq M^{1-\frac{1}{p}}\left(\sum_{i=0}^{M-1}\left\|\sum_{I \in \mathcal{E}_{i}} x_{I} \frac{h_{I}}{|I|^{1 / p}}\right\|_{L_{X}^{p}}^{p}\right)^{1 / p}
\end{aligned}
$$

it is sufficient to prove

$$
\left\|\sum_{I \in \mathcal{E}_{i}} x_{I} \frac{h_{I}}{|I|^{1 / p}}\right\|_{L_{X}^{p}} \leq c_{p}\left(\sum_{I \in \mathcal{E}_{i}}\left\|x_{I}\right\|_{X}^{p}\right)^{\frac{1}{p}} \quad \text { for } \quad i \leq M .
$$

But here we get that

$$
\left\|\sum_{I \in \mathcal{E}_{i}} x_{I} \frac{h_{I}}{|I|^{\frac{1}{p}}}\right\|_{L_{X}^{p}}
$$




$$
\begin{aligned}
& =\left(\sum_{K \in \mathcal{E}_{i}} \int_{A_{K}}\left\|\sum_{I \in \mathcal{E}_{i}} x_{I} \frac{h_{I}(t)}{|I|^{\frac{1}{p}}}\right\|_{X}^{p} d t\right)^{\frac{1}{p}} \\
& =\left(\sum_{K \in \mathcal{E}_{i}} \frac{\left|A_{K}\right|}{|K|} \int_{A_{K}}\left\|\sum_{I \in \mathcal{E}_{i}} x_{I}\left(\frac{|K|}{|I|}\right)^{\frac{1}{p}} h_{I}(t)\right\|_{X}^{p} \frac{d t}{\left|A_{K}\right|}\right)^{\frac{1}{p}} \\
& \leq\left(\sum_{K \in \mathcal{E}_{i}} \int_{A_{K}}\left\|\sum_{I \in \mathcal{E}_{i}} x_{I}\left(\frac{|K|}{|I|}\right)^{\frac{1}{p}} h_{I}(t)\right\|_{X}^{p} \frac{d t}{\left|A_{K}\right|}\right)^{\frac{1}{p}} \\
& =\left(\sum_{K \in \mathcal{E}_{i}} \int_{A_{K}}\left\|\sum_{K \subseteq I \in \mathcal{E}_{i}} x_{I}\left(\frac{|K|}{|I|}\right)^{\frac{1}{p}} h_{I}(t)\right\|_{X}^{p} \frac{d t}{\left|A_{K}\right|}\right)^{\frac{1}{p}} \\
& =\left(\sum_{K \in \mathcal{E}_{i}} \int_{A_{K}}\left\|\sum_{l=0}^{n(K)} x_{G_{-l}\left(K, \mathcal{E}_{i}\right)}\left(\frac{|K|}{\left|G_{-l}\left(K, \mathcal{E}_{i}\right)\right|}\right)^{\frac{1}{p}} h_{G_{-l}\left(K, \mathcal{E}_{i}\right)}(t)\right\|_{X}^{p} \frac{d t}{\left|A_{K}\right|}\right)^{\frac{1}{p}} \\
& =\left(\sum_{K \in \mathcal{E}_{i}} \int_{A_{K}}\left\|\sum_{l=0}^{\infty} x_{G_{-l}\left(K, \mathcal{E}_{i}\right)} \chi_{\{l \leq n(K)\}}\left(\frac{|K|}{\left|G_{-l}\left(K, \mathcal{E}_{i}\right)\right|}\right)^{\frac{1}{p}} h_{G_{-l}\left(K, \mathcal{E}_{i}\right)}(t)\right\|_{X}^{p}\right.
\end{aligned}
$$

where $G_{-l}\left(K, \mathcal{E}_{i}\right)$ form the maximal sequence of dyadic intervals from $\mathcal{E}_{i}$ such that

$$
K=G_{0}\left(K, \mathcal{E}_{i}\right) \subset G_{-1}\left(K, \mathcal{E}_{i}\right) \cdots \subset G_{-n(K)}\left(K, \mathcal{E}_{i}\right)
$$

and $G_{-n(K)}\left(K, \mathcal{E}_{i}\right)$ is the unique maximal interval in $\mathcal{E}_{i}$ containing $K$. Next we obtain an upper estimate for the last expression as follows:

$$
\begin{aligned}
& \sum_{l=0}^{\infty}\left(\sum_{K \in \mathcal{E}_{i}} \int_{A_{K}}\left\|x_{G_{-l}\left(K, \mathcal{E}_{i}\right)} \chi_{\{l \leq n(K)\}}\left(\frac{|K|}{\left|G_{-l}\left(K, \mathcal{E}_{i}\right)\right|}\right)^{\frac{1}{p}} h_{G_{-l}\left(K, \mathcal{E}_{i}\right)}(t)\right\|_{X}^{p} \frac{d t}{\left|A_{K}\right|}\right)^{\frac{1}{p}} \\
& =\sum_{l=0}^{\infty}\left(\sum_{K \in \mathcal{E}_{i}}\left\|x_{G_{-l}\left(K, \mathcal{E}_{i}\right)} \chi_{\{l \leq n(K)\}}\right\|_{X}^{p} \frac{|K|}{\left|G_{-l}\left(K, \mathcal{E}_{i}\right)\right|}\right)^{\frac{1}{p}}
\end{aligned}
$$




$$
\begin{aligned}
& =\sum_{l=0}^{\infty}\left(\sum_{\substack{I, K \in \mathcal{E}_{i} \\
G_{-l}\left(K, \mathcal{E}_{i}\right)=I}}\left\|x_{I}\right\|_{X}^{p} \frac{|K|}{|I|}\right)^{\frac{1}{p}} \\
& =\sum_{l=0}^{\infty}\left(\sum_{I \in \mathcal{E}_{i}}\left\|x_{I}\right\|_{X}^{p} \frac{\sum_{\substack{G_{-l}\left(K, \mathcal{E}_{i}\right)=I \\
\mid K}}|K|}{|I|}\right)^{\frac{1}{p}} \\
& \leq \sum_{l=0}^{\infty}\left(\sum_{I \in \mathcal{E}_{i}}\left\|x_{I}\right\|_{X}^{p} 2^{-l}\right)^{\frac{1}{p}} \\
& =\left(\sum_{l=0}^{\infty} 2^{-\frac{l}{p}}\right)\left(\sum_{I \in \mathcal{E}_{i}}\left\|x_{I}\right\|_{X}^{p}\right)^{\frac{1}{p}} .
\end{aligned}
$$

Next we turn to the case $\llbracket \mathcal{E} \rrbracket=\infty$ for which it is known that the GamlenGaudet construction yields an approximation of the Haar system by appropriate 'blocks' of $\left(h_{I}\right)_{I \in \mathcal{E}}$. The next lemma demonstrates that this construction fits perfectly with our Haar type inequalities. We carefully avoid using the unconditionality of the Haar system (and therefore the UMD property of Banach spaces) and exhibit a system of functions with exactly the same joint distribution as the ususal Haar basis, rather than to allow that the measures of the support of a Haar function and its approximation are related by uniformly bounded multiplicative constants.

Lemma 3.4. Let $X$ be a Banach space, $p \in(1,2]$, and $\mathcal{E}$ be a collection of dyadic intervals such that

$$
\llbracket \mathcal{E} \rrbracket=\infty .
$$

If there is a constant $c>0$ such that

$$
\left\|\sum_{I \in \mathcal{E}} x_{I} \frac{h_{I}}{|I|^{1 / p}}\right\|_{L_{X}^{p}} \leq c\left(\sum_{I \in \mathcal{E}}\left\|x_{I}\right\|_{X}^{p}\right)^{\frac{1}{p}}
$$

for all finitely supported families $\left(x_{I}\right)_{I \in \mathcal{E}} \subset X$, then $X$ is of Haar type $p$ with $H T_{p}(X) \leq c$.

Remark 3.5. In Lemma 3.4 the range $p \in(2, \infty)$ does not make sense since already $X=\mathbb{R}$ does not have Rademacher type $p \in(2, \infty)$ and henceforth 
Haar type $p \in(2, \infty)$. In other words, for $\llbracket \mathcal{E} \rrbracket=\infty$ and $p \in(2, \infty)$ the inequality (4) fails to be true.

Proof of Lemma 3.4. Let $n \geq 1, \delta \in(0,1)$, and $\varepsilon=2^{-n-1} \delta$. Since $\llbracket \mathcal{E} \rrbracket=\infty$ the condensation property (cf. [4, Lemma 3.1.4]) yields the existence of a $K_{0} \in \mathcal{E}$ such that

$$
\sum_{J \in G_{n}\left(K_{0}, \mathcal{E}\right)}|J| \geq(1-\varepsilon)\left|K_{0}\right| .
$$

Examining the Gamlen-Gaudet construction [2] as (for example) presented in [4, Proposition 3.1.6], we obtain a family $\left(\mathcal{B}_{I}\right)_{I \in \mathcal{D}_{n}}$ of collections of dyadic intervals such that

(i) $\mathcal{B}_{I} \subseteq K_{0} \cap \mathcal{E}$,

(ii) the elements of $\mathcal{B}_{I}$ are pairwise disjoint,

(iii) for $B_{I}:=\bigcup_{K \in \mathcal{B}_{I}} K$ one has that $B_{I} \cap B_{J}=\emptyset$ if and only if $I \cap J=\emptyset$, and $B_{I} \subseteq B_{J}$ if and only if $I \subseteq J$,

(iv) for

$$
k_{I}:=\sum_{K \in \mathcal{B}_{I}} h_{K}
$$

and $I, I^{-}, I^{+} \in \mathcal{D}_{n}$ such that $I^{-}$is the right half of $I$ and $I^{+}$the left half of $I$, one has $B_{I^{-}} \subseteq\left\{k_{I}=-1\right\}$ and $B_{I^{+}} \subseteq\left\{k_{I}=1\right\}$,

(v) for $0 \leq k \leq n$ and $|I|=2^{-k}$ one has

$$
\frac{\left|K_{0}\right|}{2^{k}}-2 \varepsilon\left|K_{0}\right| \leq\left|B_{I}\right| \leq \frac{\left|K_{0}\right|}{2^{k}} .
$$

As a consequence

$$
\frac{1-\delta}{2^{n}}\left|K_{0}\right| \leq\left|B_{I}\right| \leq \frac{\left|K_{0}\right|}{2^{n}} \quad \text { for } \quad|I|=2^{-n}
$$

and

$$
(1-\delta)\left|K_{0}\right| \leq \sum_{|I|=2^{-n}}\left|B_{I}\right| \leq\left|K_{0}\right| .
$$

Choose measurable subsets $A_{I} \subseteq B_{I}$ for $|I|=2^{-n}$ such that

(a) $\left|A_{I}\right|=(1-\delta) 2^{-n}\left|K_{0}\right|$, 
(b) the $k_{I}$ restricted to $A_{I}$ are symmetric.

Let $S:=\bigcup_{|I|=2^{-n}} A_{I}$, so that $|S|=(1-\delta)\left|K_{0}\right|$, and

$$
A_{I}:=B_{I} \cap S \text { for all (remaining) } I \in \mathcal{D}_{n} .
$$

When restricted to the probability space $\left(S, \frac{d t}{|S|}\right)$ the system $\left(k_{I}\right)_{I \in \mathcal{D}_{n}}$ has the same joint distribution as the usual Haar system $\left(h_{I}\right)_{I \in \mathcal{D}_{n}}$ on the unit interval. Hence, as a consequence of the Gamlen-Gaudet construction, we obtain that

$$
\begin{aligned}
\left\|\sum_{I \in \mathcal{D}_{n}} \frac{h_{I}}{|I|^{1 / p}} x_{I}\right\|_{L_{X}^{p}} & =\left\|\sum_{I \in \mathcal{D}_{n}} \frac{k_{I}}{|I|^{1 / p}} x_{I}\right\|_{L_{X}^{p}\left(S, \frac{d t}{S \mid}\right)} \\
& =\left(\frac{1}{|S|}\right)^{\frac{1}{p}}\left\|\sum_{I \in \mathcal{D}_{n}} \frac{k_{I}}{|I|^{1 / p}} x_{I}\right\|_{L_{X}^{p}(S, d t)} \\
& \leq\left(\frac{1}{|S|}\right)^{\frac{1}{p}}\left\|\sum_{I \in \mathcal{D}_{n}} \sum_{K \in \mathcal{B}_{I}} \frac{h_{K}}{|K|^{1 / p}}\left(\frac{|K|^{1 / p}}{|I|^{1 / p}} x_{I}\right)\right\|_{L_{X}^{p}} .
\end{aligned}
$$

Recall that we selected the collection $\mathcal{B}_{I}$ as a sub-collection of $\mathcal{E}$. Using our hypothesis concerning $\mathcal{E}$ and $X$, we obtain an upper estimate for the last term as follows,

$$
\begin{aligned}
c\left(\frac{1}{|S|}\right)^{\frac{1}{p}}\left(\sum_{I \in \mathcal{D}_{n}} \sum_{K \in \mathcal{B}_{I}} \frac{|K|}{|I|}\left\|x_{I}\right\|^{p}\right)^{\frac{1}{p}} & =c\left(\sum_{I \in \mathcal{D}_{n}} \frac{\left|B_{I}\right|}{|I||S|}\left\|x_{I}\right\|^{p}\right)^{\frac{1}{p}} \\
& =c\left(\sum_{I \in \mathcal{D}_{n}} \frac{\left|B_{I}\right|}{|I|(1-\delta)\left|K_{0}\right|}\left\|x_{I}\right\|^{p}\right)^{\frac{1}{p}} \\
& \leq \frac{c}{(1-\delta)^{\frac{1}{p}}}\left(\sum_{I \in \mathcal{D}_{n}}\left\|x_{I}\right\|^{p}\right)^{\frac{1}{p}} .
\end{aligned}
$$

Letting $\delta \downarrow 0$ yields our statement. 


\section{References}

[1] R. Deville, G. Godefroy, V. Zizler. Smoothness and renorming in Banach spaces. Longman 1993.

[2] J.L.B. Gamlen, R.J. Gaudet. On subsequences of the Haar system in $L^{p}[0,1](1 \leq p \leq \infty)$, Israel J. Math., 15 (1973), 404-413.

[3] S. Geiss, P.F. X. Müller. Extrapolation of vector valued rearrangement operators, preprint (2006).

[4] Müller, P. F. X., Isomorphisms between $H^{1}$ spaces, Birkhäuser Verlag, Basel, 2005.

[5] A. Pietsch, J. Wenzel. Orthonormal systems and Banach space geometry. Cambridge University Press, 1998.

[6] G. Pisier. Martingales with values in uniformly convex spaces. Israel J. Math. 20 (1975), 326-350.

[7] E.M. Semenov. Equivalence in $L^{p}$ of permutations of the Haar system. Dokl. Akad. Nauk SSSR, 242 (1978), 1258-1260.

\section{Addresses}

Department of Mathematics and Statistics

P.O. Box 35 (MaD)

FIN-40014 University of Jyväskylä

Finland

Department of Analysis

J. Kepler University

A-4040 Linz

Austria 